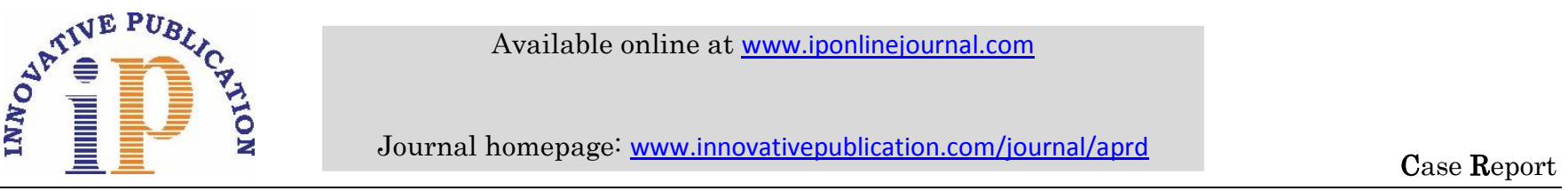

\title{
Utilising natural anterior maxillary teeth for immediate removable partial denture: A case report
}

\author{
Rini Gangwal ${ }^{1 *}$, Cerin Susan Thomas ${ }^{2}$, Divya S Sharma ${ }^{3}$ \\ ${ }^{\mathbf{1}}$ Reader, ${ }^{2}$ Post Graduate Student, ${ }^{3}$ Professor and HOD, ${ }^{\mathbf{1 - 3}}$ Dept of Pedodontics and Preventive Dentistry, ${ }^{\mathbf{1 - 3}}$ Modern Dental College and \\ Research Centre, Indore, Madhya Pradesh, India
}

\begin{abstract}
The aesthetic choice of anterior teeth is always a challenging work. Matching the size, colour, shade and shape of the restored tooth with those of the natural teeth is a dilemma. In this report patient's own natural teeth were used as a substitute in an immediate removable partial denture. Immediate replantation in aesthetic region like maxillary anterior, can hence, be considered primarily significant. The replacement of an anterior tooth is most technique sensitive, as it involves the patient's expectations, which demands artificial tooth that are identical to his/her natural tooth. Therefore, in this case presentation patient's aesthetics, function and psychological wellness was given attention.
\end{abstract}

Keywords: Aesthetics, Immediate Partial Denture, Natural teeth.

\section{Introduction}

Maxillary central incisors play a predominant role in the physical appearance and elevate the smile, aesthetics adding to the self-confidence of an individual. Utilising the crown of extracted anterior tooth as a temporary restoration can be considered as a feasible treatment option until fabrication of a definitive prosthesis. ${ }^{1}$ A biocompatible restoration of form and function can hence be ensured, thereby, preserving the original dental \& gingival contour, and simulating the architecture of neighbouring teeth.

An immediate denture is defined as a complete or removable partial denture fabricated for placement immediately after the removal of natural teeth. ${ }^{2}$ An immediate denture following extraction allows patients to continue their communal activities perpetually. The selection of appropriate artificial teeth cannot be performed at the try-in appointment owing to the reliance of patient on the clinician for its fulfilment. Nevertheless, if patient's own natural teeth can be attached to his immediate denture, the clinician can easily overcome his expectations.

The present article describes fabrication of an immediate removable partial denture using patient's own natural teeth to enhance esthetics, comfort \& self esteem to the patient.

\section{Case Report}

A 14-years old male patient was referred to the Department of Pedodontics and Preventive dentistry, with a chief complaint of discoloured teeth and pus discharge from anteriors (Fig. 1,2). His history revealed that he had a trauma 3 years back, followed by root canal treatment of 11,21 . On clinical examination, associated teeth were found to be grade II mobile, with sinus tract. Radiographically, an evidence of internal and external resorption of root was confirmed. Considering the poor prognosis of teeth, a treatment was planned to extract the affected teeth, followed by the construction of an immediate partial denture by incorporating patient's own extracted teeth, to attain better aesthetics.

\section{Clinical procedure}

1. Before extraction, Alginate impressions of maxillary and mandibular dentition were made according to the conventional technique and poured in dental stone.

2. The affected teeth $(11,21)$ were removed from the cast and were trimmed, to replicate extracted socket.

3. Teeth $11 \& 21$ were extracted (Fig. 3) under L.A and were thoroughly cleaned with saline. To control bleeding and initiate clot formation the patient was asked to bite into a gauze piece. Patient was instructed to avoid gargling and to avoid eating hot substances so as to prevent dislodgement of clot.

4. The tooth was de-coronated to the level of cementoenamel junction (Fig. 4,5). The gutta percha was removed with the help of bur. The discolouration of teeth was removed by scrapping of the discoloured dentin with slowly rotating round bur. Following this, $30 \% \mathrm{H}_{2} \mathrm{O}_{2}$ in cotton pellet was applied for 10 minutes in the pulp chamber of teeth so as to reduce the discolouration and to achieve the normal whitish hue of the teeth. Pulp chamber was thoroughly washed with water after bleaching.

5. The pulp chamber was sealed with tooth coloured composite material.

6. Using patient's own natural teeth an immediate removable partial denture was fabricated.

\footnotetext{
*Corresponding Author: Rini Gangwal, Dept. of Pedodontics and Preventive Dentistry, Modern Dental College and Research Centre, Indore, Madhya Pradesh, India 
7. After an hour, when bleeding got controlled, finished and polished immediate partial denture was placed in position (Fig. 6,7)

8. Patient was instructed about the insertion \& removal of the partial denture. Never to force prosthesis into position by biting down on it. Initally prosthesis is to be worn all the time and to be removed at night so as to allow better healing of extraction socket and moulding of alveolar bone. Patient was recalled after 24 hours for necessary adjustments and check up.

9. After 24 hours healing was uneventful \& denture retention was good. Patient was asked to brush his denture every day using soft bristled brush and mild hand soap to remove food deposits and plaque. At night the patient was asked to place the partial denture in water.

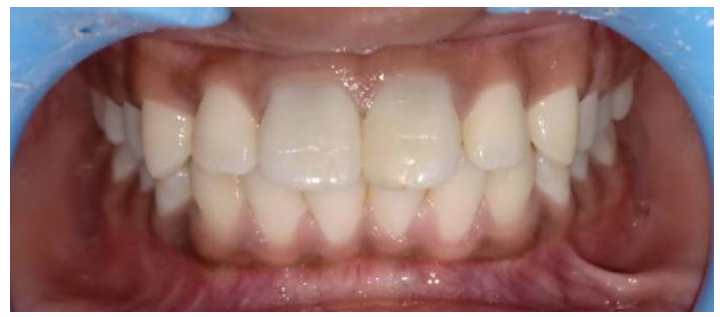

Fig. 1: Preoperative photograph showing cervical discoloration with respect to 11,21

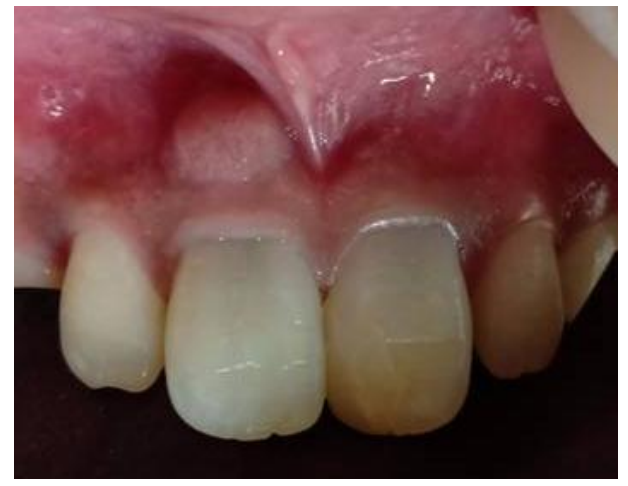

Fig. 2: Preoperative photographs showing sinus tract with respect to 11 .

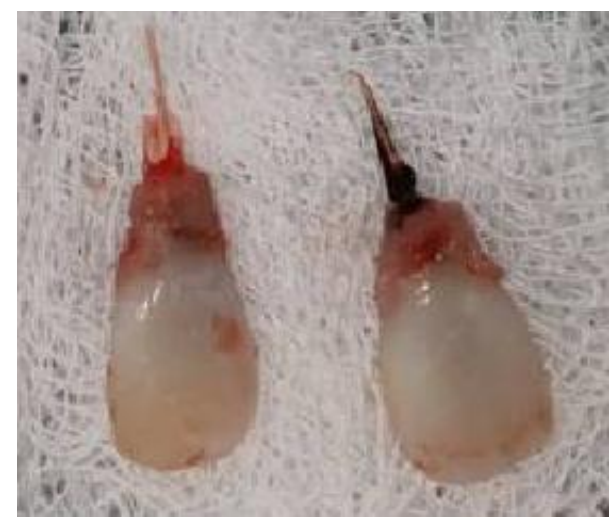

Fig. 3: Extracted 11,21 showing remaining gutta percha points.

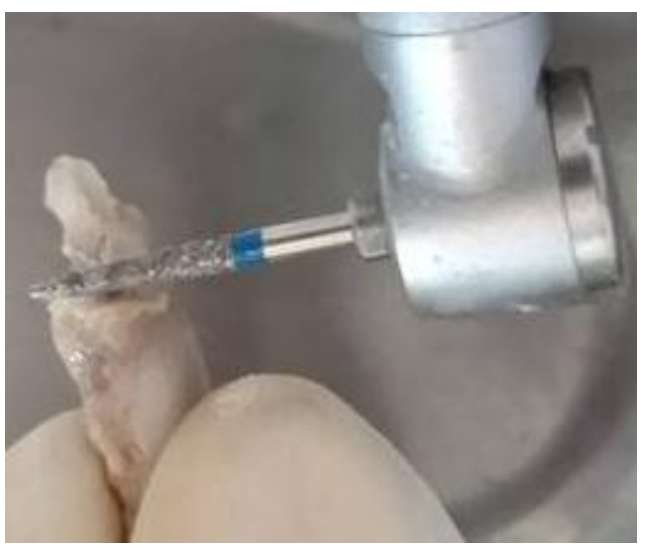

Fig. 4: Decoronation of tooth at the level of cement-enamel junction.

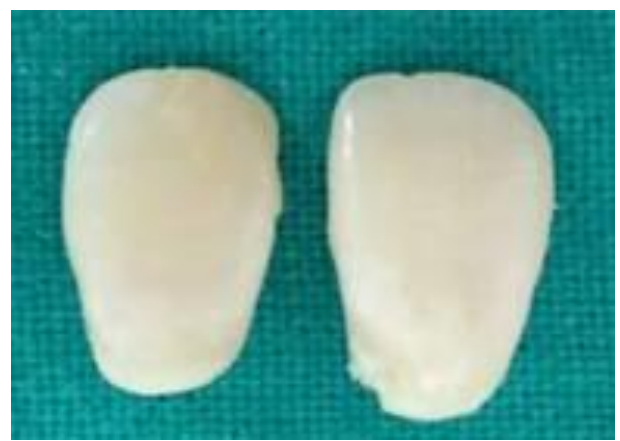

Fig. 5: Natural teeth prepared to be used as pontic.

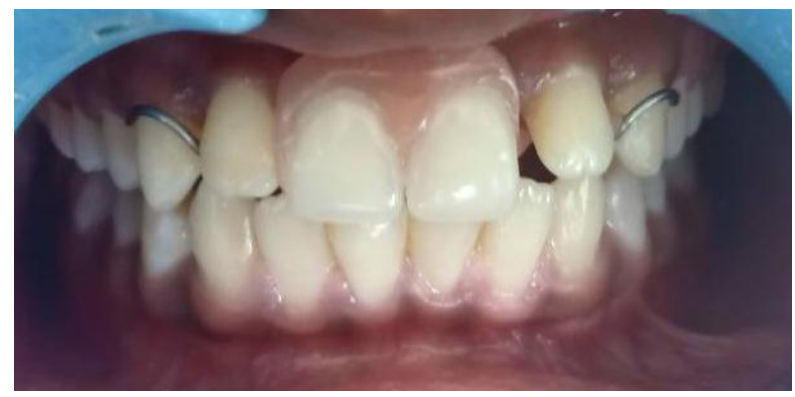

Fig. 6: Intraoral post operative photograph.

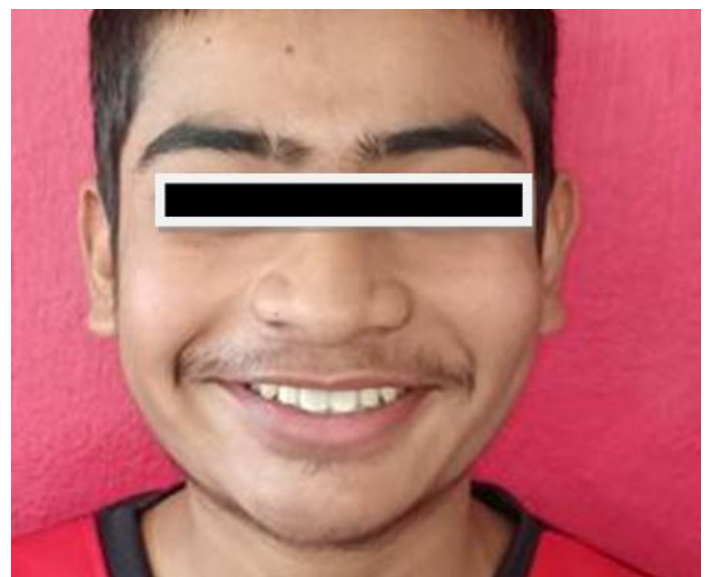

Fig. 7: Extra oral post operative photograph. 


\section{Discussion}

The prosthetic replacement of an anterior tooth is a demanding task, which include meeting the patient's expectations such as, proper colour, shape and size that should be similar to the remaining dentition. Also psychological value and technique sensitivity are of utmost importance. Although many treatment options are available for the replacement of the missing anterior tooth, the matter of concern is the patient's adaptability to the treatment. The immediate replacement techniques include: using an acrylic removable partial denture, bonding the natural tooth to the adjacent teeth by using either light cure or by means of wire bonding ${ }^{3}$. In the present report, an attempt was made by using the patients' natural teeth instead of the artificial acrylic teeth in the immediate removable partial denture. Using the natural tooth offers the benefits of being the right one with respect to size, shape and colour. Immediate denture allows excellent healing of extraction socket. This technique of making an interim restoration is economically feasible, as it requires limited laboratory support and materials, and it can be accomplished in a single appointment ${ }^{4}$. This treatment plan could serve best to the patient as per his age and needs to provide temporary aesthetic requirement till the patient turned 18 years of age for the permanent restorative alternatives.

To retain the moisture along with shade \& vital appearance of natural teeth it should not be allowed to dry. Hence for precaution for such dentures is that they must be kept in water while not in the mouth. The longevity of such denture can be enhanced by proper care. ${ }^{5}$

Lower anterior teeth would not be permitted in such cases because of inadequate retention at the cervical region also the removal of the pulpal tissue may cause undue weakening of the teeth. Similarly, this technique cannot be used for posterior teeth as replacement of their relatively large pulpal tissue with autopolymerizing resin would not be able to bear masticatory loads for long $^{6}$. It is also contraindicated in mouth breathers (natural teeth lose their vital appearance and hue due to dryness and acrylic resin may shrink), bruxers (biting forces are more than normal) and patients with neuromuscular disorders. ${ }^{7}$

\section{Conclusion}

The excellent aesthetics and patient satisfaction with immediate partial denture involving natural tooth is an appropriate treatment modality in which anterior teeth need to be replaced. A proper follow-up care is essential, for the success of immediate dentures. As we followed this approach, the patient could avoid the social stigma of appearing without teeth and he was satisfied with the final results.

\section{Source of Funding}

None.

\section{Conflict of Interest}

None.

\section{References}

1. Wasserstein J. Guided Dental Implant Surgery Using the Extracted Tooth Crown as a Temporary Restoration. Available from: http:// www.periodontist.org. [Last updated on $2010 \mathrm{Dec}$ 02].

2. Soni A. Trial anterior artificial tooth arrangement for an immediate denture patient: A clinical report. J Prosthet Dent 2000;84:260-3.

3. Srkyl A, Kadithota M. Remote tooth in immediate partial denture: a case report. J Clin Diag Res 2011; 5(6):1318-9.

4. Satapathy SK, Pillai A, Jyothi R, Annapurna PD. Natural teeth replacing artificial teeth in a partial denture: A Case Report.: $J$ Clin Diag Res 2013;7(8):1818.

5. Boucher CO. Swenson's Complete Dentures. 5th ed. St. Louis: The C.V. Mosby Company; 1964. p. 669-702.

6. Hayward DE. Use of natural upper anterior teeth in complete dentures. J Prosthet Dent 1968; 19:359-63.

7. Baslas V, Kaur S, Yadav R, Aggarwal H, Ehtisham M. Use of natural anterior maxillary teeth in immediate removable partial denture: An old technique revisited. SRM J Res Dent Sci 2016;7(3):190-3.

How to cite this article: Gangwal R, Thomas CS, Sharma DS. Utilising natural anterior maxillary teeth for immediate removable partial denture: A case report. Ann Prosthodont Restor Dent 2019;5(3):86-8. 